\title{
TITLE:
}

\section{Perioperative control of blood glucose level in cardiac surgery.}

$\operatorname{AUTHOR}(S)$ :

Minakata, Kenji; Sakata, Ryuzo

\section{CITATION:}

Minakata, Kenji ... [et al]. Perioperative control of blood glucose level in cardiac surgery.. General thoracic and cardiovascular surgery 2013, 61(2): 61-66

\section{ISSUE DATE:}

2013-02

URL:

http://hdl.handle.net/2433/189828

\section{RIGHT:}

The final publication is available at Springer via http://dx.doi.org/10.1007/s11748-0120198-9; この論文は出版社版でありません。引用の際には出版社版をご確認ご利用くだ さい。; This is not the published version. Please cite only the published version. 
Perioperative BG control in cardiac surgery. Minakata et al.

Title:

Perioperative control of blood glucose level in cardiac surgery

Kenji Minakata, MD, PhD

Ryuzo Sakata, MD, PhD

Department of Cardiovascular Surgery

Kyoto University Graduate School of Medicine

Kyoto, Japan

Running title: Perioperative glucose control in cardiac surgery

Key words: blood glucose level, cardiac surgery, diabetes mellitus, infection, mortality

Corresponding author:

Kenji Minakata, MD, Department of Cardiovascular Surgery, Kyoto University Graduate School of Medicine,

Address: 54 Kawahara-cho, Shogoin, Sakyo-ku, Kyoto, 606-8507, Japan

Telephone: +81-75-751-3780 / FAX: +81-75-751-3098

Email: minakata @kuhp.kyoto-u.ac.jp

Word count: 4,171 
Perioperative BG control in cardiac surgery. Minakata et al.

\section{Abstract (176 words)}

It is well recognized that poor perioperative blood glucose (BG) control can increase the risk of infection, cardiovascular accidents, and even death in patients undergoing cardiac surgery. Since it has been reported that tight $\mathrm{BG}$ control $(80-110 \mathrm{mg} / \mathrm{dL})$ yields better outcomes in critically ill patients, it became a standard of care to control BG using intravenous insulin infusion in ICU. However, it has been debated in terms of the optimal target range whether a strict control with intensive insulin therapy is better than liberal control. Because strict BG control can often cause hypoglycemia, which in turn increases the hospital mortality. In fact, a meta-analysis of randomized clinical trials concluded that tight BG control was not associated with significantly reduced hospital mortality but was associated with an increased risk of hypoglycemia. According to the current published guidelines, it seems to be optimal to control BG level of $140-180 \mathrm{mg} / \mathrm{dL}$ in ICU. In terms of more strict BG control (110-140 mg/dL), it may be appropriate in selected patients as long as this can be achieved without significant hypoglycemia. 
Perioperative BG control in cardiac surgery. Minakata et al.

\section{Introduction}

Physical stress induces a number of neuro-hormonal responses to maintain or increase blood glucose

(BG) level (part of the "fight or flight" response). This is especially true in cardiac surgery, which often involves one of the most invasive and stressful procedures in modern medicine: cardiopulmonary bypass. However, in diabetic patients or others with glucose intolerance, the BG level may increase beyond the optimal range, causing hyperglycemia. During or immediately after surgery, hyperglycemia can cause numerous complications: abnormal immune response, vascular malfunction, metabolic acidosis and even coma. Perhaps one of the most noteworthy complications is the increased rate of infection, including deep sternal wound infection (DSWI). It is well known that poor BG control perioperatively can increase the incidence of DSWI, which can easily lead to sepsis, multi-organ failure and even death. In this review, the dangers of perioperative hyperglycemia are discussed, and the most recent strategies for perioperative BG control are summarized based on the findings of large clinical trials.

\section{The diabetic disadvantage}

The prevalence of diabetes mellitus (DM) has increased dramatically in Western countries over the last several decades, leading in turn to increased mortality due to cardiovascular events [1]. This trend is also apparent in Asian countries, especially in Japan, where the number of DM patients has increased from 6.9 million to 8.9 million (approximately $7 \%$ of the general population) in the last 
Perioperative BG control in cardiac surgery. Minakata et al.

decade (a 29\% increase) [2]. The most important life-threatening complication in DM patients is obviously coronary artery disease [3]. There has been debate regarding the optimal treatment for DM patients with coronary artery disease. Some favor percutaneous catheter intervention (PCI), while others favor coronary artery bypass grafting (CABG). Some studies have shown that CABG yields better long-term outcomes in DM patients with multivessel disease [4, 5]. Despite the recent technological advances in PCI, such as drug-eluting stents, CABG remains the treatment of choice in many DM patients.

We recently conducted a large multi-institutional clinical study in Japanese patients undergoing isolated CABG, and reported that DM was associated with higher incidence of infection and higher mortality [6]. The overall infection rate was $9.2 \%$ in DM patients vs $6.1 \%$ in non-DM patients $(\mathrm{p}=0.036)$. Also, the hospital mortality rate was $2.1 \%$ in $\mathrm{DM}$ patients vs $1.1 \%$ in non-DM patients $(\mathrm{p}=0.124)$. These results were consistent with the others based on STS database analyses. In fact, it has been reported that DM in patients undergoing open heart surgery poses a significant risk for hospital mortality and morbidity, including stroke, DSWI and increased length of hospital stay [7]. This report found that DM patients were significantly more likely to die within 30 days following any of the cardiac procedures included in the study. In fact, the 30-day mortality in DM patients versus non-DM patients were as follows: 
Perioperative BG control in cardiac surgery. Minakata et al.

$3.96 \%$ vs $2.82 \%$ for isolated CABG; $6.63 \%$ vs $3.57 \%$ for AVR; and $11.07 \%$ vs $5.52 \%$ for MVR, respectively (all differences significant with $\mathrm{P}$ values $<0.001$ ). Likewise, the stroke rates for DM patients versus non-DM patients were as follows: $2.22 \%$ vs $1.40 \%$ for isolated CABG; $2.74 \%$ vs 1.44\% for AVR; $3.64 \%$ vs $1.94 \%$ for MVR (all differences significant with P values < 0.001) [7]. In addition, DM patients have worse long-term survival than non-DM patients after surgery [8]. The survival rate of DM patients after CABG and/or valve replacement was twice as poor as that of non-DM patients [7]. There is no doubt that DM patients have unfavorable baseline characteristics such as diffuse coronary artery disease, peripheral artery disease, high body mass index, and worse preoperative renal function, all of which could contribute to worse short- and long-term outcomes compared to non-DM patients.

\section{Deep sternal wound infection (anterior mediastinitis)}

Although surgical site infection is one of the most common post-operative complications, especially in DM patients, DSWI (anterior mediastinitis) has a profound impact on early and late morbidity and mortality. Our previous study showed that the incidence of DSWI was $2.0 \%$ in DM patients vs $1.1 \%$ in non-DM patients, which was highly likely related with increased hospital mortality [6]. Likewise, according to the STS database analyses, DM patients had nearly twice the incidence of DSWI compared to non-DM patients. Figures for specific procedures were as follows: $1.02 \%$ vs $0.45 \%$ for isolated CABG; $0.78 \%$ vs $0.41 \%$ for AVR; and $0.72 \%$ vs $0.35 \%$ for MVR (all differences significant 
Perioperative BG control in cardiac surgery. Minakata et al.

with $\mathrm{P}$ values < 0.001) [7]. DSWI definitely increases the mortality rate, as well as increasing the length of hospital stay and associated costs. In 1997, Zerr et al. reported that hyperglycemia in the first 2 postoperative days in DM patients undergoing cardiac surgery is a significant independent predictor of DSWI [9].

\section{Metabolic consequences of hyperglycemia in DM patients}

Free fatty acids are the primary energy substrate in the non-ischemic myocardium [10]. In fact, long-chain fatty acids provide more than six times as much energy as the oxidation of an equal amount of glucose. Under ischemic conditions, glucose becomes the primary energy source in the myocardium so that the ischemic myocardium can more effectively utilize the limited supply of oxygen to preserve cellular integrity and contractile function. This metabolic shift prevents the increase of toxic products of fatty acids such as oxygen free radicals and it plays an important role in myocardial and vascular preservation. In DM patients, however, glucose uptake and/or transport function is impaired, which forces the myocardium to rely heavily on free fatty acid metabolism as its primary energy source. As a result, the level of free fatty acids and their metabolism is increased, contributing to an increased level of toxic substrates leading to myocardial and vascular dysfunction. These detrimental effects are accelerated during the period of myocardial ischemia that occurs under aortic cross-clamping during cardiac surgery in DM patients. 
Perioperative BG control in cardiac surgery. Minakata et al.

\section{Effects of intravenous insulin infusion in cardiac surgery}

Several animal and human studies have reported that intravenous insulin reverses the metabolic dysfunctions associated with DM via several mechanisms, such as stimulation of myocardial glucose utilization, and anti-inflammatory/anti-oxidant effects on a cellular and molecular level [10]. This may be the reason why continuous insulin infusion, in addition to normoglycemia, improves clinical outcomes in patients with acute coronary syndrome and CABG. Lazar et al. demonstrated that BG control (serum glucose: 125-200 mg/dL) with glucose-insulin-potassium solution in patients with DM undergoing CABG improved perioperative outcomes, enhanced survival, and decreased the incidence of ischemic events and wound complications [10]. They suggested that the presence of insulin itself is important to improve the metabolism in DM patients, in addition to maintaining normal glucose levels [11].

\section{Impact of perioperative BG control in cardiac surgery}

Based on a large prospective observational study, Furnary et al. reported that there was a highly significant relationship between mortality and postoperative BG levels rising above $175 \mathrm{mg} / \mathrm{dl}$ [12].

Dr. Furnary is in charge of the Portland Diabetic Project, which is an ongoing prospective study of over 5,000 DM patients, which aims to show that tight glucose control from the end of surgery until the 2 nd postoperative day with continuous insulin infusion may eliminate the diabetic disadvantage 
Perioperative BG control in cardiac surgery. Minakata et al.

[13]. His group found that tight glucose control with a full 3 days of continuous insulin infusion (which they termed "the Portland Protocol") significantly reduced mortality (by 65\%), DSWI (by $63 \%$ ), and length of hospital stay (average 2-day reduction). They concluded that DM is not a true risk factor for the diabetic disadvantage in terms of increased mortality and morbidity when perioperative the BG level is well controlled within a certain range according to their protocol. Usefulness of perioperative BG control with intravenous insulin was also shown in patients undergoing off-pump CABG [14].

\section{How to control the BG level in a timely and effective manner with insulin:}

\section{Intermitted subcutaneous injection or continuous intravenous infusion?}

There is no doubt that insulin therapy has been the standard treatment for controlling BG in the acute clinical setting. Traditionally, regular insulin was given subcutaneously every 4-6 hours according to a certain sliding scale to control BG in the ICU or ward. In the late 1990's, however, Furnary et al. reported that continuous intravenous insulin infusion yielded better BG control than intermittent subcutaneous injection and led to a significant reduction in DSWI in patients undergoing cardiac surgery [15]. Since that time, a number of continuous insulin infusion protocols have been proposed with different target ranges in different clinical settings [16]. 
Perioperative BG control in cardiac surgery. Minakata et al.

\section{Preoperative BG control}

DM patients undergoing cardiac surgery may have different levels of diabetic control preoperatively.

As a marker of the level of BG control, hemoglobin A1c is very useful because it indicates the level

of BG for the previous 4-12 weeks. In patients where their level of hemoglobin A1c indicates poor

levels of BG control, the physician is faced with a dilemma: should he or she wait until the

hemoglobin A1c level improves before attempting surgery? Halkos et al. reported that an elevated

hemoglobin A1c level ( $\geq 7.0 \%)$ was strongly associated with adverse postoperative events such as

myocardial infarction, DSWI, renal failure and cerebrovascular incidents after CABG [17]. These

adverse events are likely due to the higher associated comorbidities such as systemic hypertension,

advanced chronic kidney disease and/or peripheral artery disease in patients with high hemoglobin

A1c. They recommended that in patients with hemoglobin A1c greater than $8.7 \%$ and not requiring

urgent or emergency $\mathrm{CABG}$, consideration should be given to achieving good BG control before

surgery to minimize morbidity and mortality. On the other hand, Furnary et al. did not identify the

level of preoperative hemoglobin A1c as an independent significant predictor of mortality, DSWI or

length of hospital stay. They only found that preoperative BG level is a risk factor for DSWI and

increased length of hospital stay. Therefore, they recommended controlling the preoperative BG

level, not the level of hemoglobin A1c. In other words, they did not recommend waiting until the

hemoglobin A1c is improved before surgery as long as BG is strictly controlled from the day of 
Perioperative BG control in cardiac surgery. Minakata et al.

surgery to the 2 nd postoperative day (three full days) with continuous intravenous insulin infusion

[13]. In general, it should be carefully screened if there are any significant indicators of poor preoperative BG control such as highly elevated HbA1c, elevated amount of urine glucose (10g/day), and presence of ketone bodies in urine in elective operations.

\section{Intraoperative BG control: Is it necessary?}

There have been a few studies on BG control during cardiac surgery. Doenst et al. reported that a high glucose level during cardiopulmonary bypass was an independent predictor of mortality in both DM and non-DM patients [18]. They also found that a high glucose level during cardiopulmonary bypass was also an independent predictor of all major adverse events in both patient groups. In addition, Ghandi et al. reported, in a retrospective observational study on 409 cardiac surgical patients, that intraoperative hyperglycemia was an independent risk factor for perioperative complications, including mortality, after adjustment for postoperative glucose concentrations [19]. Each $20 \mathrm{mg} / \mathrm{dL}$ increase in glucose concentration greater than $100 \mathrm{mg} / \mathrm{dL}$ during surgery was associated with a $34 \%$ increase in the likelihood of postoperative complications. However, they subsequently conducted the first prospective randomized study on $400 \mathrm{DM}$ and non-DM patients undergoing on-pump CABG and showed that intraoperative intensive insulin therapy with a target range of $80-100 \mathrm{mg} / \mathrm{dL}$ did not reduce perioperative mortality and morbidity, but rather increased 
Perioperative BG control in cardiac surgery. Minakata et al.

stroke rate and mortality [20]. As long as the BG level is in the liberal range ( $\leq 200 \mathrm{mg} / \mathrm{dL})$ during cardiac surgery, it may not be beneficial to attempt strict BG control. Also, caution needs to be taken to prevent profound hypoglycemia when insulin is given intravenously during anesthesia. It has been shown that attempting to maintain normoglycemia in the setting of insulin infusion may initiate postoperative hypoglycemia, potentially causing adverse events [21].

\section{The optimal target range of the BG level in the ICU: Is strict control better}

\section{than liberal control?}

The definitions of "tight," "strict" and "intensive" BG control tend to differ in each report, and it has been debated what target range is optimal. Also, it should be noted with caution that the target does not always reflect the actual level achieved. In a landmark paper, Van den Berghe et al. conducted the first prospective randomized trial comparing tight BG control (target $80-110 \mathrm{mg} / \mathrm{dL}$ ) with intensive insulin therapy to conventional BG control $(180-200 \mathrm{mg} / \mathrm{dL})$ in critically ill surgical patients [22]. This study included over 1500 patients, $63 \%$ of whom had undergone cardiac surgery before ICU admission. They found that tight BG control resulted in a significant reduction in mortality $(10.6 \%$ with intensive treatment vs $20.2 \%$ with conventional treatment, $\mathrm{p}=0.005)$ in patients who required $\geq 5$ days of ICU care with multiorgan failure and sepsis. Also, cardiac surgical mortality was reduced in those patients requiring $\geq 5$ days of ICU care with other conditions. 
Perioperative BG control in cardiac surgery. Minakata et al.

D'Alessandro et al. published a propensity analysis that showed that strict BG control significantly reduced the EuroSCORE expected mortality in DM patients undergoing CABG, especially in moderate to high-risk patients [23]. Their BG target in the ICU was $\leq 140 \mathrm{mg} / \mathrm{dl}$. In terms of long-term outcomes, Lazar et al. showed that tight perioperative glucose control (125-200 mg/dL) with glucose-insulin-potassium solution improved not only perioperative outcomes, but also long-term survival and freedom from recurrent angina comparing to the standard therapy $(<250$ $\mathrm{mg} / \mathrm{dL}$ ) [11]. Based on these studies, it is logical to conclude that it is beneficial to maintain BG at less than $180-200 \mathrm{mg} / \mathrm{dL}$ to decrease postoperative morbidity and mortality.

Of course, this begs the question: Exactly how low should the target be? Since the aforementioned landmark paper by Van den Berghe et al., it has been widely accepted that tight BG control with intensive insulin therapy $(80-110 \mathrm{mg} / \mathrm{dL})$ is better than conventional control in surgery or in the ICU. However, following this report, several randomized trials failed to show the benefit of tight BG control with intensive insulin therapy. Also, a meta-analysis of 29 randomized studies focusing on the benefits and risks of tight glucose control (very tight: $\leq 110 \mathrm{mg} / \mathrm{dL}$ or moderately tight: $<150$ $\mathrm{mg} / \mathrm{dL}$ ) in critically ill adult patients concluded that tight glucose control was not associated with significantly reduced hospital mortality but was associated with an increased risk of hypoglycemia [24]. To support these results, a recent large prospective randomized multicenter trial (the 
Perioperative BG control in cardiac surgery. Minakata et al.

NICE-SUGAR study) demonstrated that intensive BG control with a target of $81-108 \mathrm{mg} / \mathrm{dl}$

increased mortality among adults in the ICU compared with conventional BG control with a target of $180 \mathrm{mg} / \mathrm{dl}$ or less [25]. This study involved over 6,000 patients, however, the mortalities in the intensive control group and conventional control group were $27.5 \%$ and $24.9 \%$ at 90 days after randomization, respectively. In both groups, potentially life-sustaining treatments were withheld or withdrawn in more than $90 \%$ of the patients who died. Also, it seems that severe hypoglycemia commonly occurred in the intensive BG control group of the study (6.8\%), which raises the question of the safety and feasibility of the tight glucose control protocol itself. It has clearly been shown that hypoglycemia is associated with mortality in the ICU setting [26]. Because the patients in the study were so sick at the time of enrollment, it is difficult to compare the results of this study with studies on regular cardiac surgery patients, given the current acceptable mortality after CABG of around 1-2\%. Based on a large CABG cohort, Bhamidipati et al. reported that moderate glycemic control $(127-179 \mathrm{mg} / \mathrm{dL})$ was superior to tight $(\leq 126 \mathrm{mg} / \mathrm{dL})$ or liberal $(\geq 180 \mathrm{mg} / \mathrm{dL})$ glycemic control, with decreased mortality and fewer major complications [27]. They concluded that moderate control is ideal for the DM patients undergoing CABG. It may be necessary to conduct a prospective randomized study to compare tight glucose control and conventional glucose control using more sophisticated protocols with a minimum risk of hypoglycemia in exclusively cardiac surgery patients to reach a definitive conclusion. 
Perioperative BG control in cardiac surgery. Minakata et al.

\section{Is $B G$ variability more important than $B G$ level?}

Some studies have shown that variability (wide vacillation) in BG levels is more important than the achieved BG level in terms of outcomes. Egi et al., using a large multicenter cohort of patients and set of glucose measurements, found that the variability of BG was an independent predictor of ICU and hospital mortality, and that its predictive ability was greater than that of the mean BG level [28]. In addition, Hermanides et al. reported that high BG variability was closely associated with ICU and in-hospital death, and also that high BG variability combined with high mean BG values was associated with highest ICU mortality [29]. They concluded that in patients who underwent strict glycemic control, low BG variability seemed protective, even when mean BG levels remained elevated.

\section{Current published recommendations for perioperative control of BG levels}

The American Diabetes Association published guideline for the medical care of diabetes patients in 2012 [30]. According to these guidelines, in critically ill patients in the hospital, insulin therapy should be initiated for the treatment of persistent hyperglycemia starting at a threshold of no greater than $180 \mathrm{mg} / \mathrm{dL}$. Also, once insulin therapy is started, a glucose range of $140-180 \mathrm{mg} / \mathrm{dL}$ is recommended for the majority of these patients. With regard to more strict control (110-140 $\mathrm{mg} / \mathrm{dL})$, 
Perioperative BG control in cardiac surgery. Minakata et al.

it may be appropriate in selected patients as long as this can be achieved without significant hypoglycemia.

The STS guidelines recommend that patients with and without diabetes with persistently elevated serum glucose $(\geq 180 \mathrm{mg} / \mathrm{dL})$ should receive IV insulin infusions to maintain serum glucose $\leq 180$ $\mathrm{mg} / \mathrm{dL}$ for the duration of their ICU care. All patients who require three or more days in the ICU because of ventilator dependency, the need for inotropes, intra-aortic balloon pumps, left ventricular assist device support, antiarrhythmics, dialysis, or continuous veno-venous hemofiltration, should receive a continuous insulin infusion to keep $\mathrm{BG}$ at $\leq 150 \mathrm{mg} / \mathrm{dL}$, regardless of their diabetic status. Also, before intravenous insulin infusion is discontinued, patients should be transitioned to a subcutaneous insulin schedule using institutional protocols [31]. The American College of Physicians Guidelines also recommends a target BG level of 140 to $200 \mathrm{mg} / \mathrm{dL}$ in medical/surgical ICU patients with hyperglycemia [32]. It does not recommend intensive insulin therapy because of the high likely hood of hypoglycemia.

\section{Conclusions}


Perioperative BG control in cardiac surgery. Minakata et al.

In conclusion, hyperglycemia during and/or after cardiac surgery entails several risks, such as an increased risk of infection, stroke, and myocardial or vascular dysfunction. Therefore, BG levels should be maintained at $140-180 \mathrm{mg} / \mathrm{dL}$ with continuous insulin infusion. 
Perioperative BG control in cardiac surgery. Minakata et al.

\section{References}

1) Bonow RO, Gheorghiade M. The diabetes epidemic: a national and global crisis. Am J Med 2004; 116 Suppl 5A:2S-10S.

2) Annual report from the national survey of life and nutrition 2007, the Ministry of Health, Labour and Welfare of Japan (Japanese). Available at http://www.mhlw.go.jp/houdou/2008/12/h1225-5.html

3) Flaherty JD, Davidson CJ. Diabetes and coronary revascularization. JAMA 2005; 293: 1501-8.

4) Brener SJ, Lytle BW, Casserly IP, Schneider JP, Topol EJ, Lauer MS. Propensity analysis of long-term survival after surgical or percutaneous revascularization in patients with multivessel coronary artery disease and high-risk features. Circulation 2004; 109: 2290-5.

5) Hlatky MA, Boothroyd DB, Bravata DM, Boersma E, Booth J, Brooks MM, et al. Coronary artery bypass surgery compared with percutaneous coronary interventions for multivessel disease: a collaborative analysis of individual patient data from ten randomised trials. Lancet 2009; 373:1190-7.

6) Minakata K, Bando K, Takanashi S, Konishi H, Miyamoto Y, Ueshima K, et al. Impact of Diabetes Mellitus on Outcomes in Japanese Patients Undergoing Coronary Artery Bypass Grafting. Journal of Cardiology 2012; 59: 275-284

7) Brown JR, Edwards FH, O'Connor GT, Ross CS, Furnary AP. The diabetic disadvantage: historical outcomes measures in diabetic patients undergoing cardiac surgery - the preintravenous insulin era. Smin Thorac Cardiovasc Surg 2006: 18; 281-288

8) Szabó Z, Håkanson E, Svedjeholm R. Early postoperative outcome and medium-term survival in 540 diabetic and 2239 nondiabetic patients undergoing coronary artery bypass grafting. Ann Thorac Surg 2002; 74:712-9.

9) Zerr KJ, Furnary AP, Grunkemeier GL, Bookin S, Kanhere V, Starr A. Glucose control lowers the risk of wound infection in diabetics after open heart operations. Ann Thorac Surg 1997; 63: $356-361$.

10) Lazar HL. Alterations in myocardial metabolism in the diabetic myocardium. Sem Thorac Cardiovasc Surg 2006;18:289-292

11) Lazar HL, Chipkin SR, Fitzgerald CA, Bao Y, Cabral H, Apstein CS. Tight glycemic control in diabetic coronary artery bypass graft patients improves perioperative outcomes and decreases recurrent ischemic events. Circulation 2004; 109: 1497-502.

12) Furnary AP, Gao G, Grunkemeier GL, Wu Y, Zerr KJ, Bookin SO, et al. Continuous insulin infusion reduces mortality in patients with diabetes undergoing coronary artery bypass grafting. J Thorac Cardiovasc Surg 2003; 125: 1007-21.

13) Furnary AP, Wu Y. Eliminating the diabetic disadvantage: The Portland Diabetic Project. Semin Thorac Cardiovasc Surg 2006; 18:302-8. 
Perioperative BG control in cardiac surgery. Minakata et al.

14) Fujii T, Watanabe $Y$, Shiono N, Kawasaki M, Yokomuro H, Ozawa T, et al. Usefulness of perioperative blood glucose control in patients undergoing off-pump coronary artery bypass grafting. Gen Thorac Cardiovasc Surg 2007; 55: 409-15

15) Furnary AP, Zerr KJ, Grunkemeier GL, Starr A. Continuous intravenous insulin infusion reduces the incidence of deep sternal wound infection in diabetic patients after cardiac surgical procedures. Ann Thorac Surg 1999; 67: 352-60.

16) Kelly JL, Hirsch IB, Furnary AP. Implementing an intravenous insulin protocol in your practice: practical advice to overcome clinical, administrative, and financial barriers. Semin Thorac Cardiovasc Surg 2006; 18: 346-358

17) Halkos ME, Puskas JD, Lattouf OM, Kilgo P, Kerendi F, Song HK, et al. Elevated preoperative hemoglobin A1c level is predictive of adverse events after coronary artery bypass surgery. $\mathrm{J}$ Thorac Cardiovasc Surg 2008; 136: 631 - 640.

18) Doenst T, Wijeysundera D, Karkouti K, Zechner C, Maganti M, Rao V, et al. Hyperglycemia during cardiopulmonary bypass is an independent risk factor for mortality in patients undergoing cardiac surgery. J Thorac Cardiovasc Surg 2005; 130:1144.

19) Gandhi GY, Nuttall GA, Abel MD, Mullany CJ, Schaff HV, Williams BA, et al. Intraoperative hyperglycemia and perioperative outcomes in cardiac surgery patients. Mayo Clin Proc 2005; 80: 862-6.

20) Gandhi GY, Nuttall GA, Abel MD, Mullany CJ, Schaff HV, O'Brien PC, et al. Intensive intraoperative insulin therapy versus conventional glucose management during cardiac surgery: a randomized trial. Ann Intern Med 2007; 146: 233-43.

21) Chaney MA, Nikolov MP, Blakeman BP, Bakhos M. Attempting to maintain normoglycemia during cardiopulmonary bypass with insulin may initiate postoperative hypoglycemia. Anesth Analg 1999; 89: 1091-5

22) Van den Berghe G, Wouters P, Weekers F, Verwaest C, Bruyninckx F, Schetz M, et al. Intensive insulin therapy in the critically ill patients. N Engl J Med 2001; 345:1359-67.

23) D'Alessandro C, Leprince P, Golmard JL, Ouattara A, Aubert S, Pavie A, et al. Strict glycemic control reduces EuroSCORE expected mortality in diabetic patients undergoing myocardial revascularization. J Thorac Cardiovasc Surg 2007; 134:29-37.

24) Wiener RS, Wiener DC, Larson RJ. Benefits and risks of tight glucose control in critically ill adults: a meta-analysis. JAMA 2008; 300: 933-44.

25) NICE-SUGAR Study Investigators, Finfer S, Chittock DR, Su SY, Blair D, Foster D, Dhingra V, et al. Intensive versus conventional glucose control in critically ill patients. N Engl J Med 2009; 360: 1283-97.

26) Hermanides J, Bosman RJ, Vriesendorp TM, Dotsch R, Rosendaal FR, Zandstra DF, et al. 
Perioperative BG control in cardiac surgery. Minakata et al.

Hypoglycemia is associated with intensive care unit mortality. Crit Care Med. 2010; 38: $1430-4$.

27) Bhamidipati CM, LaPar DJ, Stukenborg GJ, Morrison CC, Kern JA, Kron IL, et al. Superiority of moderate control of hyperglycemia to tight control in patients undergoing coronary artery bypass grafting. J Thorac Cardiovasc Surg 2011; 141: 543-51.

28) Egi M, Bellomo R, Stachowski E, French CJ, Hart G. Variability of Blood Glucose Concentration and Short-term Mortality in Critically Ill Patients. Anesthesiology 2006; 105:244-52

29) Hermanides J, Vriesendorp TM, Bosman RJ, Zandstra DF, Hoekstra JB, Devries JH. Glucose variability is associated with intensive care unit mortality. Crit Care Med. 2010; 38: 838-42.

30) American Diabetes Association. Executive summary: Standards of medical care in diabetes 2012. Diabetes Care 2012; 35 Suppl 1:S4-S10.

31) Lazar HL, McDonnell M, Chipkin SR, Furnary AP, Engelman RM, Sadhu AR, et al. The Society of Thoracic Surgeons Practice Guideline Series: Blood glucose management during adult cardiac surgery. Ann Thorac Surg 2009; 87: 663-9.

32) Qaseem A, Humphrey LL, Chou R, Snow V, Shekelle P: the clinical Guidelines Committee of the American College of Physicians. Use of intensive insulin therapy for the management of glycemic control in hospitalized patients: a clinical practice guideline from the American College of Physicians. Ann Intern Med. 2011; 154: 260-7. 